Cahiers de Narratologie

Analyse et théorie narratives

Les avatars du chapitre en bande dessinée

\title{
La tomaison des albums de Buddy Longway a-t-elle valeur de chapitrage?
}

Alain Corbellari

\section{(2) OpenEdition}

Journals

Electronic version

URL: http://journals.openedition.org/narratologie/9048

DOI: 10.4000/narratologie.9048

ISSN: 1765-307X

Publisher

LIRCES

\section{Electronic reference}

Alain Corbellari, «La tomaison des albums de Buddy Longway a-t-elle valeur de chapitrage ? », Cahiers de Narratologie [Online], 34 | 2018, Online since 08 January 2019, connection on 14 November 2019. URL : http://journals.openedition.org/narratologie/9048; DOI : 10.4000/narratologie.9048

This text was automatically generated on 14 November 2019.

Article L.111-1 du Code de la propriété intellectuelle. 


\title{
La tomaison des albums de Buddy Longway a-t-elle valeur de chapitrage?
}

\author{
Alain Corbellari
}

1 La série Buddy Longway de Derib s'est très vite signalée dans le monde de la bande dessinée francophone par un trait original qu'elle reste encore aujourd'hui l'une des rares à avoir vraiment assumé : le vieillissement de son héros au fil des épisodes.

2 L'on sera amené, à se demander ici dans quelle mesure Derib avait prémédité les conséquences de cette innovation, mais une chose est certaine: il ne pouvait pas ignorer qu'il avait transgressé dès le premier épisode l'une des principales règles implicites régissant le monde des séries dessinées, à savoir le célibat de ses protagonistes. Le modèle disneyen, certes, flanquait volontiers le héros d'une éternelle fiancée (Minnie pour Mickey, Daisy pour Donald), mais celle-ci lui était donnée a priori et l'on avait vite compris que cette relation ne pouvait en aucun cas évoluer vers un authentique mariage, d'autant plus qu'à la fiancée venaient s'ajouter des neveux dont l'âge était fixé une fois pour toutes. Dans la BD francophone, ce type de relations n'est pas non plus inconnu: songeons aux couples jamais complètement officialisés que forment Gaston Lagaffe et Mademoiselle Jeanne ou Natacha et Walter. En passant, notons qu'il n'est peut-être pas indifférent que le dessinateur qui a récemment voulu inventer la rencontre de Minnie et de Mickey soit un Suisse, et qui plus est un disciple de Derib, je veux parler de Cosey et de son charmant one-shot Une mystérieuse mélodie ou Comment Mickey rencontra Minnie ${ }^{1}$.

3 Mais le premier épisode de Buddy Longway, Chinook, prépublié dès 1972 dans Tintin et paru en album en $1974^{2}$, ne nous raconte pas seulement la rencontre du trappeur Buddy et de l'Indienne Chinook; il se conclut sur leur mariage ${ }^{3}$. Ce trait que l'on a presque envie de dire d'embourgeoisement (et que d'aucuns ont pu trouver éminemment helvète !) tranche alors sur tout ce que l'on a pu voir dans le monde des séries BD, car, dans le cours de la même aventure, il fait passer le héros d'un statut à un autre radicalement différent. De surcroît, si, avec les beaux sentiments qu'il professe, Derib 
exalte la pureté de l'amour de Buddy et de Chinook, ce n'est certainement pas pour nous montrer le héros papillonner dans les albums suivants. Et de fait, jusqu'à l'ultime album, la parfaite fidélité des deux époux l'un à l'autre ne sera jamais prise en défaut.

On peut donc dire que la nécessité de faire vieillir Buddy Longway était déjà virtuellement contenue dans sa première aventure, comme préalable à la continuation même de la série. À la fin du deuxième album (L'Ennemi), Chinook annonce à Buddy qu'elle est enceinte, instaurant avec l'album suivant une forme peu conventionnelle de suspense, le lecteur étant amené à se demander quel sera le sexe et surtout le destin de l'enfant. Jérémie naît donc dans le troisième album (Trois hommes sont passés), et on le voit dès les trois premières pages atteindre l'âge de sept ans, qui définira le chronotope de la nouvelle aventure, lequel ne changera d'ailleurs quasiment pas dans les albums 4 (Seul) et 5 (Le Secret). Nouveau saut chronologique dans l'album 6 (L'Orignal), où l'on fait la connaissance de Kathleen, la sœur cadette de Jérémie, qui y a à peu près cinq ans et que l'on peut donc considérer comme ayant approximativement dix ans de moins que son frère. Si l'on admet qu'elle a dix-huit ans dans l'ultime album de la série, on peut donc en conclure que l'ensemble des vingt albums recouvre grosso modo une durée de trente ans (2 jusqu'à la naissance de Jérémie, 10 jusqu'à la naissance de Kathleen, et 18 jusqu'à la majorité de celle-ci). On fera remarquer en passant que ce chiffre de deux enfants est singulièrement faible pour un couple vivant si près de la nature: à l'évidence le modèle de Derib est la cellule familiale petite-bourgeoise de l'Europe moderne plus que la tribu des pionniers du XIX ${ }^{\mathrm{e}}$ siècle, mais nous nous éloignons de notre sujet.

5 Buddy Longway n'est certes pas tout à fait la seule série BD francophone à faire vieillir son héros : avant lui, Giraud, pour qui Derib n'a jamais caché son admiration et son amitié ${ }^{4}$, avait certes déjà fait évoluer les traits de Blueberry, et, au moment où paraît Chinook, il y a déjà beau temps que le fringant lieutenant de Fort Navajo (premier album de la série en 1965) s'est buriné. Cependant, l'album de Blueberry à partir duquel la suite des aventures du héros de Giraud devient vraiment continue n'est que le $13^{\mathrm{e}}$ (Chihuaha Pearl), qui est presque exactement contemporain de Chinook. Certes, les albums 7 (Le Cheval de fer) à 10 (Général Tête-Jaune) de Blueberry avaient déjà auparavant formé une unité (à laquelle la suite de la saga finira par se raccorder en mettant inopinément Tête-Jaune à la tête du complot contre Grant), mais on peut soutenir que ce type de structuration résultait davantage de la fuite en avant à laquelle son imagination débordante conduisait le scénariste Charlier que d'une volonté consciente de voir le héros vieillir. D'ailleurs, en l'absence de repères temporels précis, comme ceux que Derib pose en montrant l'évolution de la famille de son héros, le vieillissement de Blueberry, dans la série principale ${ }^{5}$, reste, si l'on ose dire, essentiellement empirique.

6 Le cas de Thorgal est plus complexe: postérieur à Buddy Longway (ses premières aventures ne paraissent dans Tintin qu'à partir de 1977), le héros de Rosinski (qui, soit dit en passant, vit depuis longtemps en Valais !) a sans doute été influencé par Derib, dont il reprend le concept d'une évolution basée sur la croissance d'une cellule familiale : comme Buddy, Thorgal a d'Aaricia deux enfants, d'abord un garçon, Jolan, puis une fille, Louve, mais l'évolution de la série ne présente en rien la solidité structurelle de Buddy Longway: multiplication des aventures adventices, invraisemblable stabilité physionomique du couple Thorgal-Aaricia, rebondissements de plus en plus capillotractés qui, outre d'improbables résurrections de personnages, nous montrent un Thorgal faisant un enfant à Kriss de Valnor, après avoir, il est vrai, 
perdu la mémoire, car la fidélité de Thorgal à Aaricia - poncif nécessité par les impératifs moraux que se donne la BD pour adolescents - est aussi exemplaire que celle de Buddy à Chinook. À l'évidence, la série Thorgal, qui a de surcroît vu se multiplier les séries parallèles, a été victime de son succès et a reculé devant la solution définitive de Derib qui a tout bonnement assassiné son héros dans le $20^{\mathrm{e}}$ (et donc définitivement dernier!) épisode de Buddy Longway, preuve que le dessinateur suisse était suffisamment sûr de ses capacités à renouveler son inspiration pour oser ce sacrifice commercialement peut-être coûteux.

7 Cette liquidation du héros apparaît au demeurant chez Derib comme la conséquence logique du coup de force sur lequel se concluait le premier tome : au courage de marier Buddy Longway a correspondu - fait sans exemple dans le monde itératif par excellence des séries BD - celui de narrer sa mort, et celle de son épouse dans le dernier tome d'une série, dont il est temps d'examiner maintenant plus en détail la construction.

8 La première chose qu'il convient de préciser est que l'évolution temporelle du héros n'est pas ipso facto liée à l'enchaînement narratif des albums. Jusqu'à son $10^{\mathrm{e}}$ tome, soit très exactement dans toute sa première moitié, le série des Buddy Longway, en dépit de quelques rappels (procédé dont toutes les séries BD sont familières - avec des notes du type "voir Buddy Longway à New York») est constitué d'épisodes se suffisant à euxmêmes. Dans un premier temps, Derib n'a donc pas vu de problème à se conformer, du moins en apparence, à un schéma classique qui pouvait assurer la pérennité ad libitum de la série, et ce d'autant plus qu'il lui était loisible d'en figer arbitrairement le chronotope : comme nous l'avons vu, les épisodes 3 à 5 nous montrent un Jérémie ayant en gros le même âge. Ils ne pourraient cependant pas être intervertis sans dommage, car le volume $\mathrm{n}^{\circ} 5$ s'ouvre sur un Buddy dont la jambe plâtrée est la conséquence direct de ses mésaventures du $4^{\mathrm{e}}$ épisode (Seul) et, surtout, se termine sur un événement traumatisant, à savoir l'incendie de la cabane des Longway par la main criminelle du méchant Indien Cheval Fougueux. (Signalons déjà pour ménager le suspense, que l'on n'a pas fini d'entendre parler de cet Indien indigne et déséquilibré.)

Cet incendie constitue le premier grand élément disrupteur de la série qui, comme on l'a déjà signalé, fait soudain un saut de plusieurs années jusqu'au seuil de l'album $n^{\circ} 6$, où l'équilibre de la série se trouve de fait modifié par l'arrivée de Kathleen. En conséquence, Jérémie s'y retrouve être un adolescent d'une quatorzaine d'années fin prêt pour des aventures en solitaire, dont le tome 5 donnait d'ailleurs un avant-goût, puisque suite à l'accident de Buddy, le centre d'intérêt de l'intrigue se déplaçait déjà sur l'héritier présomptif. Cependant, en même temps qu'il illustre une rupture, le $6^{\mathrm{e}}$ album inaugure une nouvelle stase, et les enfants Longway vont garder la même physionomie jusqu'au $9^{e}$ tome, Premières chasses, qui est même essentiellement constitué, comme son titre l'indique, de la reprise, habilement agencée à travers des récits rétrospectifs faits à Kathleen, de précédentes aventures plus brèves. Y figure même la première " histoire complète ", antérieure à Chinook, de Buddy Longway, dont le graphisme semi-caricatural, typique de la première manière de Derib, jure franchement avec un style réaliste qui s'est de plus en plus affirmé au cours de l'évolution de la série ${ }^{6}$.

Les choses changent à nouveau avec Le Démon blanc, $10^{\mathrm{e}}$ album, non que le temps écoulé depuis le précédent tome soit si important (deux ans peut-être ?), mais parce que c'est dans cette aventure que va véritablement se mettre en place l'engrenage qui fera de chacun des volumes suivants la conséquence directe du précédent, et comme les 
chapitres successifs d'un seul et même roman ${ }^{7}$. Cet album, peut-être le plus beau de la série, nous raconte l'épreuve initiatique vécue par un Jérémie de 16-17 ans qui, suite à un jeûne ascétique de quelques jours en pleine nature, voit se dessiner dans la forme d'un nuage la silhouette d'un cheval blanc qu'il va se mettre en devoir de trouver pour le mater. Entre-temps, contrepoint presque anodin à cette trame principale, Buddy, dont le cheval est mort dans les premières pages de l'album, se rend au fort proche, dont il connaît les habitants depuis l'album $n^{\circ} 4$, mais où il a la désagréable surprise de tomber nez-à-nez avec un nouveau personnage, l'antipathique officier de cavalerie Ryan, qui voit d'un mauvais œil qu'il vive "avec une squaw » et qui se retrouve fort vexé qu'un cheval qu'il convoite soit finalement offert à Buddy par son ami Michael, tenancier du fort.

11 Or, les deux fils narratifs vont se rejoindre à la fin de l'album : Jérémie, qui a trouvé et dompté son "démon blanc", se voit dépossédé de son triomphe par l'irruption du capitaine Ryan et de ses hommes, qui se livrent à un abominable carnage sur le troupeau de chevaux sauvages au sein de laquelle Jérémie évoluait en totale communion avec la nature. Traitant les soldats de «lâches ", il se fait rudoyer par l'un d'entre eux et en conçoit dès lors une haine inexpiable pour ceux qui maltraitent ses frères rouges. L'aventure est apparemment close, mais on sent bien que ce finale dysphorique est gros de menaces. De fait, pour la première fois, Buddy et sa famille sont confrontés à un danger que l'on pourrait dire structurel: leurs relations avec les Indiens n'ont jusque là été que ponctuellement troublées (dans la mesure où ils parvenaient à échapper aux luttes claniques ou à des personnages déviants comme Cheval Fougueux), et les Blancs qu'ils ont croisé soit sont devenus des amis (comme les gens du Fort), soit ne les ont jamais trop gravement menacés (les trois desperados de Trois hommes sont passés, n'ont, comme le dit le titre de l'album, justement fait que " passer »). Avec Ryan, c'est le sens de l'Histoire qui se révèle soudain à Buddy. Jusque là, la série était assez difficile à dater, et peut-être Derib n'avait-il guère songé, avant ce dixième tome, à situer précisément les aventures de son trappeur sans reproches dans la grande Histoire. Pour la première fois, dans Le Démon blanc, un élément solide de datation apparaît dans l'uniforme et le shako à pompon du Capitaine Ryan, typique de la guerre du Mexique, ce qui nous place donc assez précisément dix à quinze ans avant la Guerre de Sécession, c'est-à-dire vers les années 1845-1850. Conteur humaniste et volontiers manichéen, Derib dévoile ici son attachement viscéral et inconditionnel pour la culture des Indiens d'Amérique du Nord, et sa condamnation absolue du génocide perpétré par les Blancs à partir de la seconde moitié du XIX ${ }^{\mathrm{e}}$ siècle. Nous sommes donc ici à l'orée de cette période noire et il semble bien que, puisque, dans la grande Histoire, les Blancs n'ont laissé aucune chance aux Indiens, Derib va se sentir dès lors contraint de faire évoluer les aventures de Buddy Longway vers une issue tragique. En a-t-il déjà conscience lorsqu'il inscrit le mot fin à la dernière page du Démon blanc? Ce qui est sûr, c'est que cette nécessité va se faire de plus en plus clairement jour dans les volumes suivants.

Le $11^{\mathrm{e}}$ album ( $\mathrm{L} a$ Vengeance) commence en apparence comme une aventure aussi nouvelle et dépourvue d'attaches que les précédentes, mais le voyage qu'y entreprend Buddy va inaugurer une errance qui ne se résoudra que dans le $16^{\mathrm{e}}$ tome (Le Dernier Rendez-vous) et influera directement sur les quatre derniers. Michael a disparu, Buddy se rend donc avec Jérémie à Bear Town, où il a la surprise de retrouver l'un des trois bandits de Trois hommes sont passé, Curly (sosie de Cosey !8) repenti et devenu un honorable tenancier de saloon, qui sera désormais l'un de ses plus fidèles alliés. Après 
avoir délivré Michael des griffes d'un dangereux illuminé9, Buddy se verra contraint de laisser Jérémie, blessé dans l'aventure, à Bear Town et de revenir seul vers Chinook et Kathleen.

13 Et c'est dans le tome 12 que le drame va devenir irréversible : retrouvant sa cabane occupée par des gens qu'il ne connaît pas, Buddy apprend de la bouche du capitaine Ryan que sa «squaw » et sa fille ont été chassées. Ryan mourra à la fin de l'épisode, mais Buddy ne retrouvera Chinook et Kathleen, on l'a dit, que quatre albums plus tard. Quant à Jérémie, dont l'absence avait déjà privé Buddy d'un appui essentiel en ces moments critiques, on ne le retrouvera qu'au tome 18 (La Balle perdue)! les tomes 13, 14, 15 et 16 vont donc narrer la longue quête de Buddy, que Derib va entrecouper de deux grands flash-backs : le premier, dans le tome 13 (Le Vent sauvage) va nous raconter ce que le lecteur aurait au fond aimé savoir depuis le tout premier tome, à savoir comment Chinook avait été faite prisonnière par les deux trafiquants dont Buddy la délivrait à l'aube de ses aventures, non sans se demander dans un premier temps ce qui lui avait pris de délivrer une squaw: à l'initiation amoureuse se superposait donc, dans ce premier tome, l'ouverture au monde des Indiens. Le second flash-back, dans le tome 16, nous racontera les aventures du père de Buddy, Harold Longway, de sa traumatisante émigration en Amérique (puisque son épouse meurt sur le bateau, face à un Buddy tout enfant) à son adoption dans une tribu indienne et à sa mort tragique.

Entre-temps, dans le tome 13, Buddy fera la connaissance de l'émigrant Gregor et de sa femme, qui ne le quitteront pratiquement plus jusqu'à la fin de ses aventures. Les tomes 13 à 16 sont donc construits sur les étapes successives d'une reconstruction personnelle et relationnelle qui aboutira à ses retrouvailles avec Chinook et Kathleen, recueillies par une tribu indienne, mais pas avec Jérémie.

Cette dernière aporie appelait sans aucun doute une suite, mais après le tome 16 de Buddy Longway, Derib va s'accorder une longue pause : de 1974 à $1987^{10}$, les albums s'étaient succédé à la cadence rapide d'un, voire deux albums par an. Il faudra en revanche attendre quinze ans, soit jusqu'en 2002, pour voir enfin paraître le $n^{\circ} 17$ (Regarde au-dessus des nuages). Entre-temps Derib, qui avait déjà publié de 1983 à 1985, en trois volumes d'une justesse parfaite, l'histoire de Celui qui est né deux fois, personnage d'indien à peu près contemporain de Buddy, aura approfondi son amour des Indiens d'Amérique en narrant, en quatre tome, de 1988 à 1998, Red Road, un récit plutôt mélodramatique centré sur la vie des Indiens d'aujourd'hui abrutis dans des réserves infamantes ${ }^{11}$.

16 Le tome 17 de Buddy Longway, en 2002, s'ouvre sur un long résumé, agrémenté de nombreuses images emblématiques, des 16 premiers tomes, résumé rendu nécessaire par le souci d'informer une nouvelle génération de lecteurs, et qui, du même coup, permet d'insister sur la cohérence narrative de l'ensemble de la série. Les fils narratifs sont repris avec une grande rigueur : le tome 17 met en scène la fille du capitaine Ryan qui meurt, prise à son propre piège en tentant de venger son père. On suit ensuite le soldat qui était le compagnon de la jeune fille, mais qui ne parvient pas davantage à éliminer la famille Longway, et c'est en même au cours de ce $18^{\mathrm{e}}$ épisode (La Balle perdue) que Buddy retrouve enfin Jérémie, découvrant non sans malaise que celui-ci est devenu le chef d'une bande d'Indiens tueurs de Blancs. Une sorte de justice immanente, sanctionnant ce demi-acquiescement à ce que l'on pourrait appeler son " côté obscur ", fera que Jérémie meurt à la fin de l'album. Le $19^{\mathrm{e}}$ épisode (Révolte) nous confronte à un trafiquant non sans rapport avec ceux que l'on rencontrait dans l'album n ${ }^{\circ} 8$ (L'Eau-de- 
feu). Enfin, le $20^{\mathrm{e}}$ tome (La Source), sorti en 2006, nous narre la mort de Slim le Borgne lors d'une chasse à l'ours (qui graphiquement rappelle un épisode du $\mathrm{n}^{\circ} 9$, Premières chasses) avant de faire revenir Cheval Fougueux, qui va assassiner Buddy et Chinook. Un épilogue nous montre Kathleen se recueillant sur la tombe de ses parents avec son mari Jeff (rencontré dans l'album $n^{\circ} 19$ ) et se promettant de continuer leur combat en faveur des Indiens, mais avec d'autres armes, puisqu'elle habite désormais «à la ville et a entrepris des études de droit. À tous égards le cycle est clos: les trois principaux protagonistes sont morts et la dernière survivante de la famille Longway prend congé de l'Ouest sauvage.

On pourrait pointer quelques menus problèmes chronologiques: Slim le Borgne a (nonobstant l'évolution graphique propre de Derib) tout à fait la même dégaine dans le premier et le dernier album, alors que trente ans de diégèse les séparent, et César, le père de Michael est déjà complètement chenu dans le $n^{\circ} 4$, ce qui ne l'empêche pas d'intervenir encore dans le $20^{e}$. On chipotera éventuellement aussi sur l'excellente forme de Buddy, qui est un quinquagénaire remarquablement alerte au moment où il meurt, et sur la bonne santé de quelques Indiens qui parcourent également toute la série $^{12}$. C'est là en vérité bien peu de choses, surtout si on les met en regard de la cohérence à la fois des détails et de l'ensemble de la trame, que Derib n'a d'ailleurs eu de cesse de consolider au fur et à mesure de l'écriture des albums. Trois tomes, au milieu de la série, les $n^{\circ} 10$ à 12 , forment en particulier un nœud structurel qui en gouverne les lignes de force, autour du capitaine Ryan, personnification, on l'a dit, de la mécanique broyeuse de l'Histoire en marche, qui apparaît dans le dixième tome et meurt dans le douzième. Emblématiqument, sa mort est la première d'une série de disparitions qui vont progressivement endeuiller toute la série. Celle-ci n'avait certes pas hésité à faire mourir certains personnages, ainsi des vilains trappeurs qui avaient capturé Chinook dans le tome 1, ou des sordides trafiquants d'alcool du tome 8, mais aucune de ces morts n'avait eu de répercussion sur l'évolution de la série. Il en va autrement de Ryan qui, tout méchant qu'il est, a droit à une agonie les yeux ouverts, fixés sur le ciel, comme dans les rituels funèbres des Indiens, et dont la disparition suscitera un long cycle de vengeances. À cet égard le titre du n 11 , La Vengeance (même si c'est contre Michael et non contre Buddy que celle-ci s'exerce), a aussi une valeur de présage, annonçant le motif essentiel des actions néfastes qui entacheront les derniers épisodes. C'est également dans le $\mathrm{n}^{\circ} 12$ que l'on note la récurrence d'un motif dramatique qui terminait le $\mathrm{n}^{\circ} 5$, à savoir l'incendie, pour la deuxième fois, de la cabane des Longway. Or, c'est précisément l'incendiaire du tome 5, Cheval Fougueux, qui, estimant visiblement que sa vengeance n'était pas complète, sera l'assassin de Buddy et Chinook dans le tome 20 ! On pourrait peut-être dénoncer ici une facilité narrative, car ce deuxième acte de vendetta, vingt ans après le premier, peut apparaître faiblement motivé, mais la réintroduction d'un personnage perdu de vue permet de renforcer l'unité de l'ensemble en forçant le lecteur à réinterpréter a posteriori l'apparente autonomie structurelle des premiers épisodes de la série.

On pourrait raffiner l'analyse de détail des albums de Buddy Longway, mais, la place nous étant comptée, on se permettra de considérer que les éléments ici dégagés suffisent à corroborer l'hypothèse qui a guidé mon analyse : nous avons bien affaire ici à un récit complet, idéalement clos et constitué de 20 épisodes, que j'ose en l'occurrence comparer à des "chapitres" romanesques, concourant tous à l'unité structurale de l'ensemble. Sauf erreur, cette complétude est unique dans la BD francophone: Blueberry et Thorgal, que j'ai invoqués en comparaison, sont très loin 
d'atteindre à ce degré de cohérence : le refus des scénaristes de tuer la poule aux œufs d'or, en différant sans cesse la mort du héros, y reconduit, malgré d'indéniables efforts de constructions narratives et chronologiques évolutives, ce que l'on pourrait appeler la malédiction sérielle des séries $\mathrm{BD}$, la dilution et la surenchère dans le rebondissement finissant par en compromettre fatalement les effets de linéarité. On pourrait en dire de même du Jonathan de Cosey, héros sur le retour qui multiplie, à mon sens un peu fâcheusement, les aventures sentimentales (ou assimilées) dans les derniers tomes parus de la série ${ }^{13} \ldots$

Certes, la plupart des séries connaissent un accroissement progressif de leur personnel et de leurs topoï narratifs, qui peut donner l'illusion d'une évolution chronologique de l'ensemble. Mais, pour prendre un exemple utra-canonique, en dépit de l'introduction successive des Dupondt, d'Haddock, de Tournesol et de la Castafiore dans les aventures de Tintin, celui-ci aura toujours 20 ans et ni le finale carnavalesque des Picaros, ni même la monumentalisation ironique du protagoniste dans L'Alph'Art n'avaient en soi de vocation authentiquement conclusive ${ }^{14}$.

L'utilisation plutôt parcimonieuse que j'ai faite jusqu'à maintenant de la notion qui est l'enjeu du présent volume, m'est ici suggérée par une analogie directe avec la technique de construction du roman dans son acception la plus traditionnelle de long récit clos sur lui-même. Je ne tenterai certes pas de proposer une définition du chapitre en littérature: les types en diffèrent du tout au tout d'un romancier à l'autre ${ }^{15}$. Mais je retiendrai tout de même l'idée que les chapitres de romans peuvent remplir au moins deux fonctions, en apparence contradictoires, d'ouverture et de clôture : ils permettent de relier et/ou d'isoler. Ils relient lorsque leurs derniers mots font espérer une suite; c'est généralement le cas (mais pas seulement) des feuilletons et de la littérature à suspense. Ils isolent lorsque les romanciers privilégient un découpage en "scènes ", propension que les noms de Flaubert, de Dostoïveski ou de Thomas Mann suffisent à illustrer. Enfin, dans bien des cas, ces deux fonctions alternent ou se complètent dans les mêmes ouvrages ${ }^{16}$.

Or, c'est, me semble-t-il, exactement ce que l'on observe dans le « roman » (graphique) de Buddy Longway, où la logique épisodique, plus prononcée dans les 10 premiers tomes, n'apparaît pas contradictoire avec une évolution chronologique concertée de l'ensemble et avec des rappels de motifs, de personnages et d'aventures qui densifient le tout et construisent sa cohérence interne. Plus exactement, si c'est bien la clôture finale (la mort du protagoniste) qui nous permet la réinterprétation après coup des premiers épisodes comme faisant indubituablement partie d'un tout, cette unité n'est pas pour autant arbitraire. Derib avait en effet déjà disposé entre les premiers épisodes des jalons de continuité suffisamment clairs pour que l'unité finale soit ressentie comme organique. Mais encore une fois, il fallait cette clôture pour que la continuité cesse d'être contingente et devienne nécessité.

De fait, les vingt «chapitres » de la série observent presque tous une unité thématique et narrative qui les individualise, mais dont les effets d'attente sont loin d'être bannis. À la fin de Chinook, le mariage de Buddy est à la fois conclusion et ouverture sur un type nouveau d'aventures, où le héros ne sera plus seul à construire son quotidien. À la fin de L'Ennemi, la promesse de la naissance d'un enfant suscite la curiosité du lecteur quant à l'avenir de la famille. À la fin du Secret, l'incendie de la cabane laisse espérer une reprise en main de leur destin par Buddy et les siens ; et de fait, l'épisode suivant, en faisant état de la naissance, entre-temps, de Kathleen, viendra apporter une réponse 
espérée, quoique pas forcément attendue, à cette situation de détresse présumée. À la fin du Démon blanc, l'intervention de l'armée fait peser une évidente menace sur la tranquillité de nos héros. À la fin de La Vengeance, le simple fait que Jérémie reste en convalescence à Bear Town introduit une incertitude sur le retour de Buddy, mais ce sera au début du volume suivant qu'interviendra le véritable coup de théâtre: l'annonce de la disparition de Chinook et de Kathleen. Et Le Dernier Rendez-vous, pour prendre un ultime exemple, commencera de manière très inattendue par une scène maritime nous ramenant quelque quarante ans en arrière, au moment de la petite enfance de Buddy. Or, ce flash-back que rien ne laissait soupçonner va déboucher, à la fin de l'épisode, sur les retrouvailles du héros avec sa femme et sa fille, absentes depuis quatre albums, même si un autre flash-back, sur Chinook, dans Le Vent sauvage, était venu entre-temps adoucir quelque peu la dureté de cette absence.

Ici encore, on pourrait multiplier les remarques mettant en évidence la double fonction - fermante et ouvrante - des 20 unités de la saga de Buddy Longway. Qu'il me suffise, pour moi aussi clore et ouvrir mon propos, de constater que la machine narrative élaborée durant 44 ans par Derib autour de son trappeur fétiche présente, à mes yeux, toutes les caractéristiques d'un roman fleuve, dont les vingt livraisons échelonnées représentent d'autant mieux les chapitres successifs que la technique graphique de notre auteur privilégie des unités de longueur raisonnable. Alors que les scénarios d'un Charlier (pour proposer une dernière comparaison avec Blueberry) sont si denses que chacun de ses albums a, à soi tout seul, des allures de roman complexe, les volumes de Derib ne développent que des intrigues assez dépouillées, condensées en scènes clés amoureusement décomposées à travers un art hors du commun de la mise en pages signifiante ${ }^{17}$. Cette pratique épurée me semble, ici encore, davantage assimilable à la notion de "chapitre» que nombre de scénarios à tiroirs dont foisonne la BD francophone. Cela n'est bien sûr pas un jugement de valeurs, mais j'espère avoir ainsi mis en exergue la profonde originalité d'un ensemble narratif dont l'exégèse mérite à coup sûr de nouveaux développements.

\section{BIBLIOGRAPHY}

Baetens, Jan (1998), Formes et politique de la bande dessinée, Louvain, Peeters.

Chessex, Jacques (1982), Judas le Transparent, Paris, Grasset.

Corbellari, Alain (2016), « Derib et le gaufrier éclaté », Bédéphile, n², p. 44-53.

Derib (Claude De Ribaupierre) (1974-2006), Buddy Longway, Bruxelles, Editions du Lombard, 20 volumes.

Derib (Claude De Ribaupierre) (1983-1985), Celui qui est né deux fois, Bruxelles, Editions du Lombard, 3 volumes.

Derib (Claude De Ribaupierre) (1988-1998), Red Road ${ }^{18}$, Bruxelles, Editions du Lombard, 4 volumes. 
Derib (Claude De Ribaupierre) \& Georges Pernin (1981), L'Aventure d'une BD, Bruxelles, Le Lombard.

Dionne, Hugo (2008), La Voix aux chapitres, Paris, Seuil.

\section{APPENDIXES}

Les 20 albums de Buddy Longway : quelques repères structurels

Chinook : rencontre et mariage de Buddy et de Chinook / apparition de slim le Borgne

L'Ennemi

Trois hommes sont passés : naissance de Jérémie / apparition de Curly

Seul : apparition de César, Nancy et Michael

Le Secret : apparition de Cheval Fougueux / $1^{\mathrm{er}}$ incendie de la cabane

L'Orignal : naissance de Kathleen

L'hiver des chevaux

L'eau-de-feu

Premières chasses : flash-backs sur Buddy

Le Démon blanc : apparition de Ryan

La Vengeance : réapparition de Curly

Capitaine Ryan : Jérémie laissé à Bear Town / disparition de Kathleen et Chinook / $2^{\mathrm{e}}$ incendie de la cabane / mort de Ryan

Le Vent sauvage : flash-back sur Chinook / apparition de Gregor

La Robe noire

Hooka-Hey

Le dernier rendez-vous : flash-back sur le père de Buddy / réapparition de Kathleen et chinook

Regarde au-dessus des nuages : apparition et mort de la fille de Ryan

La Balle perdue : réapparition et mort de Jérémie

Révolte

La Source : mort de Slim le Borgne / réapparition de Cheval Fougueux / assassinat de Buddy et de Chinook / Kathleen quitte la Frontière pour aller se marier et étudier en ville

\section{NOTES}

1. Voir aussi par ailleurs, dans ce même volume, la communicaiton d'Anaïs Goumand. 
2. Je n'entrerai pas ici en matière sur le passage de la publication fragmentée en hebdomadaire à la reprise en album, la publication périodique ne me paraissant qu'une contingence qui n'interfère nullement avec la méthode de travail de Derib.

3. Comme me le rappelle Alain Boillat, nombre d'éléments diégétiques de Buddy Longway dénotent la nette influence du western crépusculaire de Sidmey Pollack, Jeremiah Johnson (1972).

4. C'est en effet sous son réel patronyme, et avec sa propre physionomie, que le créateur de Blueberry apparaît, on aimerait dire en chair et en os, comme personnage de Buddy Longway, à partir du $8^{\mathrm{e}}$ tome de la série. Derib glisse par ailleurs un autre clin d'œil à Giraud en imaginant, dans le tome 11, la rencontre de Buddy et de... Jim McClure! et j'ai montré ailleurs (Corbellari 2016) que la séquence de la lutte de l'Indien avec l'aigle dans le tome 10 (Le Démon blanc) de Buddy Longway constituait un hommage évident à Giraud, par reprise d'une séquence du $21^{\mathrm{e}}$ tome des aventures de Blueberry (Nez cassé).

5. Il est vrai que la saga de Blueberry est compliquée par la parution parallèle des albums de La Jeunesse de Blueberry, puis par la reprise de la série par d'autres scénaristes après le décès de Charlier.

6. Même son humour tranche avec la série telle qu'elle a évolué : le jeune Buddy y est par exemple montré prêt à attaquer un ours avec son petit couteau, sous le prétexte que de grands chasseurs n'ont pas hésité à user d'une arme aussi modeste. Constatant que l'ours est décidément très menaçant, Buddy finit par s'enfuir en se donnant pour excuse qu « il devait s'agir d'ours plus petits », plaisanterie qui nous rapproche davantage de l'esprit de Yakari que de celui de Buddy Longway.

7. Est-ce un hasard si c'est précisément cet album qui sert à illustrer la fabrication d'un ouvrage de Derib dans L'Aventure d'une BD de Derib et Pernin (1981) ? De fait, cet album marque bel et bien le moment où Derib prend conscience des potentialités romanesques de sa série.

8. Cosey se serait-il formalisé d'avoir servi de modèle à un personnage peu recommandable dans Trois hommes sont passés? Toujours est-il que dans le $8^{\mathrm{e}}$ tome de son Jonathan, Le Privilège du serpent, il prêtera les traits de Derib au personnage assez louche du médecin hâbleur et charlatan Casimir Forel!

9. Ce personnage de chrétien fondamentaliste et pervers s'inscrit assez bien dans le paysage religieux de l'Amérique des pionniers, bien que ce soit là un type assez peu exploité dans le western traditionnel. De fait, ce n'est sans doute pas un hasard si on le voit décrit par un dessinateur vaudois, la Suisse romande étant fort familière de ce genre d'individus. Coïncidence étonnante : l'année même où paraît La Vengeance (1982), Jacques Chessex publie chez Grasset l'un de ses romans les plus sulfureux, Judas le transparent, qui met en scène une secte préfigurant celle du Temple solaire (Chessex se vantera, lors du massacre prépétré par celle-ci en 1994, de son don de divination!) installée en plein pays de Vaud.

10. C'est à dire jusqu'à la disparition du journal Tintin, ce qui montre bien le caractère presque fortuit de cette interruption.

11. Remarquons en passant que la réédition en un seul volume, et sous le titre englobant de la seconde, de l'ensemble de ces deux séries montre bien l'insatisfaction que Derib ressentait face à la contrainte de l'album traditionnel. En un temps où le roman graphique émergeait à peine, Celui qui est né deux fois et Red Road illustrent clairement la volonté de Derib de casser les cadres narratifs. Toute l'évolution finale de Buddy Longway ne fait que corroborer cette propension.

12. Par contre, la vieillissement de l'Indienne qui a aimé le père de Buddy, dans le tome 16, est si marqué qu'il paraît exagéré par rapport à la temporalité de la série.

13. Et l'on ne parlera pas des «cycles» dont le succès a suscité des suites souvent intempestives : les quatre merveilleux premiers albums de Balade au bout du monde, magnifique histoire complète qui ne réclamait vraiment aucune continuation, se sont ainsi vus «complétés » par des cycles supplémentaires passablement pitoyables. 
14. On pourrait étendre l'enquête aux séries de gags en une planche, qui ne fonctionnent au fond pas différemment des séries à histoires complètes. Le cas de Gaston Lagaffe peut ainsi apparaître troublant, car Gaston a spectaculairement évolué, en 20 ans, du fainéant benêt à l'écologiste conscientisé. Mais, en l'occurrence, des trente glorieuses affairistes, dans lesquelles la résistance au système passait par le sabotage, au post-modernisme désenchanté, au sein duquel la révolte se traduit par le combat humanitaire, ce n'est pas Gaston, toujours aussi jeune après 20 ans, qui a changé, mais le Monde lui-même ! Et on trouverait aussi des cas nombreux de dessinateurs qui trichent, le meilleur exemple, à cet égard, étant peut-être suisse (ce qui nous ramène d'une certaine manière au tropisme deribien): Titeuf voit ainsi son père passer tour à tour par le chômage et la dépression, et assiste surtout à la naissance de sa petite sœur, sans grandir d'un pouce!

15. Si tant est que ceux-ci usent de cet élément structurel ; ainsi ni Balzac ni Proust ne divisentils leurs romans en chapitres.

16. Voir Baetens, 1998, p. 98 et Dionne, 2008, p. 443.

17. Je me permets de renvoyer, à ce propos, à mon article "Derib et le gaufrier éclaté " (Corbellari 2016), où j'analyse la grammaire graphico-narrative de Derib et où je montre, sur l'exemple d'une séquence imitée de Giraud (en manière d'hommage) que pour raconter la même chose que l'auteur de Blueberry, celui de Buddy Longway a besoin de deux fois plus de place. À pagination égale, un album de Giraud sera donc narrativement beaucoup plus dense qu'un album de Derib.

18. Le même titre a présidé à la réédition en un seul volume de toute la série augmenté de Celui qui est né deux fois, chez le même éditeur, en 2007.

\section{ABSTRACTS}

The twenty albums of Derib's Buddy Longway series, published from 1974 to 2006, present a narrative coherence and a diegetic continuity without example to this degree in French comics. By choosing to kill his hero in the last volume, Derib closes his series without the possibility of prolonging it and incites the reader to reinterpret the global narrative as a single "novel" whose twenty albums would be like successive chapters. We analyze here the modalities and the consequences of this long-term narrative construction.

Les vingt albums de la série Buddy Longway de Derib, édités de 1974 à 2006, présentent une cohérence narrative et une continuité diégétique sans exemple à ce degré dans la bande dessinée francophone. En choisissant de tuer son héros dans le dernier tome, Derib clôt sa série sans possibilité de la prolonger et incite le lecteur à réinterpréter l'ensemble narratif qu'elle forme comme un seul "roman» dont les vingt albums seraient comme les chapitres successifs. On analyse ici les modalités et les conséquences de cette construction narrative au long cours.

\section{INDEX}

Mots-clés: bande dessinée, série, technique romanesque, personnages récurrents, Derib, Buddy Longway

Keywords: comics, series, novelistic techniques, recurring characters, Derib, Buddy Longway 


\section{AUTHOR}

\section{ALAIN CORBELLARI}

Alain Corbellari, né en 1967 à La Chaux-de-Fonds, est professeur de littérature française médiévale aux Université de Lausanne et de Neuchâtel. Spécialiste de l'histoire des études médiévales et en particulier de la figure de Joseph Bédier, il est membre du Groupe de recherches sur l'Histoire de la Philologie romane, dirigé par Michel Zink et rattaché au Collège de France et a écrit des ouvrages sur la littérature cléricale du Moyen Age, sur le Guillaume d'Orange épique, sur Romain Rolland, Ernest Renan et Charles Albert Cingria, à la nouvelle édition des CEuvres complètes de qui il participe activement. L'un de ses principaux axes de recherches est la réception de la culture médiévale dans la modernité, en particulier dans la bande dessinée, sujet sur lequel il a dirigé deux volumes collectifs, dans Etudes de Lettres en 2001 (dans la foulée d'une exposition organisée à la Bibliothèque municipale de Lausanne avec le Prof. Alexander Schwarz et Cuno Affolter) et aux Editions InFolio en 2014 (actes, publiés avec Aurélie Reusser-Elzingre, d'un colloque co-organisé à Orléans avec le Prof. Bernard Ribémont). Il a publié de nombreux articles sur les rapports du Moyen Age et de la bande dessinée et a proposé plusieurs conférences et ateliers sur ce sujet. Il travaille actuellement sur une formalisation des procédés comiques de Goscinny, Franquin et Mandryka. 